\title{
Studies of open-loop pointing in the presence of induced motion
}

\author{
ROBERT B. POST \\ University of California, Davis, California \\ and \\ ROBERT B. WELCH \\ NASA Ames Research Center, Moffett Field, California
}

\begin{abstract}
In the present research, we examined the influence of induced motion (IM) on open-loop pointing responses and the possibility that IM alters the registration of either eye or trunk position. In two experiments, subjects tracked a dot that oscillated vertically while a rectangular stimulus oscillated horizontally. The pairing of frame and dot motion caused the dot to appear to move on a slant, due to IM. In the first experiment, the subjects made judgments of the apparent slant of the dot's motion and, on separate trials, pointed open loop at the apparent location of the dot at the endpoints of its motion. Both responses were influenced by IM, although the effect on dot localization was less than the amount predicted by the IM, as indicated by the slant responses. Results were similar immediately following IM and after a 5-sec delay. In the second experiment, the subjects pointed open loop either at the apparent location of the endpoints of the tracked dot's motion or at the apparent location of one of three other briefly flashed stationary dots. The pointing responses directed toward the fixated IM target were influenced by IM to a greater extent than the responses directed toward the stationary dots. The results of the two experiments are inconsistent with the hypothesis that the effect of IM on open-loop pointing at the IM target results completely from altered perception of either eye or trunk position, since misregistration of either would be expected to influence, in a similar fashion, pointing at both the tracked dot and the briefly flashed, stationary targets.
\end{abstract}

Induced motion (IM) is the illusory perceived movement of visual stimuli in the direction opposite the real motion of other stimuli (e.g., Duncker, 1929). Attempts to point with an unseen limb (open-loop pointing) at a target undergoing IM err in the direction of the perceived motion, but by considerably less than the IM would predict (Abrams \& Landgraf, 1990; Bacon, Gordon, \& Schulman, 1982; Bridgeman, Kirch, \& Sperling, 1981). That is, real target motion of the same duration and velocity as that experienced during IM would result in greater displacement than is shown when IM targets are pointed at.

The noncommensurability between amount of seen motion and pointing errors in the IM situation has been interpreted by Bridgeman et al. (1981) as evidence that IM affects visual appearances (the cognitive system) but has relatively less influence on motor actions (the motor system). Thus, they described this as an example of visionaction dissociation (e.g., Milner \& Goodale, 1995). A similar explanation has been offered for a variety of other illusions, including size contrast illusions (Aglioti, DeSouza, \& Goodale, 1995; Haffenden \& Goodale, 1998), the

We are grateful to Steve Wade for extensive assistance in display programming and data collection. Correspondence concerning this article should be addressed to R. B. Post, Department of Psychology, University of California, Davis, CA 95616 (e-mail: rbpost@ucdavis.edu).
Müller-Lyer illusion (Gentilucci, Chieffi, Daprati, Saetti, \& Toni, 1996; Wraga, Creem, \& Proffitt, 2000), the misperception of depth intervals (Loomis, Da Silva, Fujita, \& Fukusima, 1992), overestimates of hill pitch (Creem \& Proffitt, 1998), the Dietzel-Roelofs effect (Bridgeman, Peery, \& Anand, 1997), and misperception of eye level in pitched visual environments (Welch \& Post, 1996). In each instance, despite the presence of a demonstrable visual illusion, motor responses are relatively accurate. Recently, Glover and Dixon (2001) have recast the apparent dissociation in terms of the relative contributions of mechanisms involved in the planning and the control of action. Within this conceptualization, illusions affect the planning of movement but do not influence its on-line control.

Also contrary to the vision-action dissociation hypothesis, Abrams and Landgraf (1990) and Smeets and Brenner (1995) have argued that with IM, the dissociation is not between cognitive and motor systems, but between the perceived velocity of the stimulus, which is influenced by the motion of other stimuli (hence, IM), and the egocentric location of the stimulus, which is not. Support for this conceptualization has been provided by Proteau and Masson (1997), who found that moving a background influenced subjects' attempts to displace a cursor to a target's location. The results were consistent with the hypothesis that the background motion affected the perceived velocity of the cursor, rather than the perceived location of the 
target. Within this velocity-location dissociation conceptualization, the question is not why the effects of IM on open-loop pointing are incommensurate with seen motion but, rather, why IM should have any effect at all on a localization measure (e.g., open-loop pointing).

One hypothesis concerning how IM might alter egocentric localization measures, such as open-loop pointing, is that the motion of the inducing stimulus causes subjects to systematically err when trying to fixate the IM target, which in turn leads to pointing errors. This possibility, although consistent with subjects' claims that they are ocularly tracking the IM target (e.g., Duncker, 1929; Mack, Fendrich, \& Wong, 1982), may be rejected because several studies have reported that fixation of an IM target is accurate and, thus, follows its physical, rather than its perceptual, path (Bassili \& Farber, 1977; Brosgole, Cristal, \& Carpenter, 1968; Mack et al., 1982).

An alternate hypothesis is that IM causes misregistration of eye position so that, despite accurate target fixation, subjects misperceive the direction of their gaze and point (open loop) accordingly (McConkie \& Farber, 1979; Rock, Auster, Schiffman, \& Wheeler, 1980). This proposal is consistent with the findings of Soechting, Engel, and Flanders (2001), who found a high correlation between errors of gaze and errors in pointing while IM displays were viewed and concluded that gaze information strongly influences pointing. However, Mack, Heuer, Fendrich, Vilardi, and Chambers (1985) argued against this possibility on the basis of their finding that subjects are able to accurately direct their eyes to auditory targets following IM, whereas they err in the direction of the IM when attempting to look at the apparent straight-ahead. They reasoned that if the IM display causes misperception of eye position, saccades to an auditory target would be inaccurate by an amount equal to the gaze direction misregistration. Failing to obtain this result, they concluded that pointing errors engendered by IM do not reflect altered egocentric localization of the IM target but, rather, a response bias to point in the direction of the previously seen IM in an attempt to maintain consistency between pointing and perceived motion. This, then, is not a perceptual event but a cognitive one, similar perhaps to the visual straightahead shift described by Harris (1974) in the context of prism adaptation.

A third possibility is that movement of the inducing stimulus induces a shift of perceived trunk orientation in the same direction as the stimulus motion. If the trunk has been perceived to rotate in the direction of the inducing stimulus motion, attempts to point open loop at the IM target would be aimed in the direction opposite the perceived trunk rotation, which would result in the reported errors in pointing in the same direction as seen IM. This hypothesis would also be consistent with findings that the apparent straight-ahead shifts in the direction of the inducing stimulus motion (Brosgole, 1968; Mack, Heuer, Fendrich, et al., 1985). The finding that saccades are accurately directed to auditory targets following IM (Mack, Heuer,
Fendrich, et al., 1985) does not bear on this hypothesis, because auditory targets are localized, at least initially, in head-centric, rather than body-centric, coordinates. ${ }^{1}$ To our knowledge, no previous research has examined the hypothesis that errors in open-loop pointing directed to IM targets originate with a change in perceived trunk orientation.

The present research explored further the nature of openloop pointing errors following IM. A vector sum IM stimulus was used, in which the vertical oscillation of a target was phase-locked to the horizontal oscillation of a surrounding frame. In this situation, the real vertical motion of the dot combines with the illusory horizontal IM vector to yield apparent motion on a slant (Gogel \& MacCracken, 1979; Wallach, Bacon, \& Schulman, 1978). The degree of apparent slant is a measure of the strength of the IM. This type of display has not been used in previous studies of open-loop pointing in the presence of IM. It has the potential advantage that attempts to point at the endpoints of dot motion must necessarily be directed at different spatial loci, because of the real vertical motion of the stimulus. In studies in which the IM stimulus is stationary, subjects may be less likely to point in different loci than if the target's location (in the vertical dimension) actually shifts.

In Experiment 1, the subjects judged the apparent slope of the path that a dot traveled within a horizontally oscillating frame and pointed open loop at the apparent location of the endpoints of its path. In addition, the subjects made open-loop pointing responses and apparent slope judgments for targets that actually moved on variously slanted paths. Their responses on these measures were then used to calibrate the data for the IM target. This procedure obviated the possibility that any apparent differences in the effects of the IM display on seen motion and perceived location were due merely to an inability to accurately match slope or point open loop at targets. A final factor involved taking measures either immediately after viewing the IM display or after a delay of $5 \mathrm{sec}$. The delay condition was included because of the observation that motor responses directed toward displays containing size illusions often display a greater apparent effect of the size illusion if a delay is included prior to the response. For example, open-loop pointing at the endpoints of the MüllerLyer figure is accurate or nearly accurate, despite the presence of the length illusion, when the figure is concurrently viewed (Mack, Heuer, Villardi, \& Chambers, 1985) but is much less accurate (in the direction of the illusion) if pointing is delayed (Elliot \& Lee, 1995; Mack, Heuer, Villardi, \& Chambers, 1985). ${ }^{2}$ Similarly, grasping responses directed toward Müller-Lyer figures display a greater illusion effect following a delay (Westwood, McEachern, \& Roy, 2001), as do grasping responses directed at objects that contain a relative size difference $(\mathrm{Hu} \&$ Goodale, 2000). Finally, the Dietzel-Roelofs effect influences openloop pointing in only some subjects with recently extinguished targets but influences pointing for all subjects following a brief delay (Bridgeman et al., 1997). On the basis of these previous reports, we reasoned that imposing a 
delay before allowing subjects to point at IM targets might enhance the effect of the IM and make these responses more commensurate with the amount of seen motion.

In Experiment 2, pointing measures were again obtained for the dot stimulus and, in addition, for other targets that were presented individually for brief periods following IM. We predicted that if IM causes misregistration of trunk orientation, pointing responses to any visual stimulus would err in the direction of the immediately preceding IM, and by the same amount as that measured for the IM stimulus dot. Alternately, if there is no effect of IM on trunk registration, pointing responses for other visual targets should be accurate. This technique also should be sensitive to any misregistration of gaze direction and, therefore, provides a test formally similar to that of Mack, Heuer, Fendrich, et al. (1985) for potential gaze misregistration. Unlike that work, however, the present studies employed (1) open-loop reaching rather than saccadic movements and (2) visual rather than auditory targets.

\section{EXPERIMENT 1}

\section{Method}

Subjects. Ten volunteers ( 3 males and 7 females), 19 to 45 years of age, served as subjects. All the subjects had normal vision and ei- ther were able to focus at the distance of the displays without correction or could do so with contact lenses. Two subjects were familiar with the experimental hypotheses.

Apparatus. The apparatus is depicted schematically in Figure 1. The subjects were seated and viewed the stimulus displays by looking into a box containing a mirror that was rotated $45^{\circ}$ about the vertical axis with respect to their line of sight. The stimulus displays were rear-projected onto the semitranslucent left wall of the box, which, because of the mirror, appeared to the subjects to be straight ahead of them. The subjects could reach under the mirror with a pen and place marks on a sheet of paper mounted on the rear wall of the box at the apparent location of various display features. Both the distance from their eyes to the rear wall of the box and the optical path from the eyes to the displays were $45 \mathrm{~cm} .^{3}$

The stimulus display is depicted schematically in Figure 2. The central dot was produced by a laser and was approximately $0.3 \mathrm{~cm}$ in diameter. The surrounding rectangle, which was projected by a slide projector, had $1-\mathrm{cm}$-wide sides and subtended $9 \mathrm{~cm}$ vertically $\times 15 \mathrm{~cm}$ horizontally (outer dimensions). The laser spot and the rectangle were reflected from mirrors mounted on galvanometers, so that the two stimuli could be oscillated vertically and horizontally, respectively. A microcomputer with digital-to-analog circuitry and amplifiers supplied the voltages to drive the galvanometers. The motions of the spot and the rectangle were phase-locked so that upward and downward movements of the spot were paired with rightward and leftward movements of the rectangle, respectively. The motions of both the dot and the rectangle were sinusoidal at $0.1 \mathrm{~Hz}$, with a peak-to-peak amplitude of $5.2 \mathrm{~cm}\left(6.6^{\circ}\right)$. Shutters under microcom-
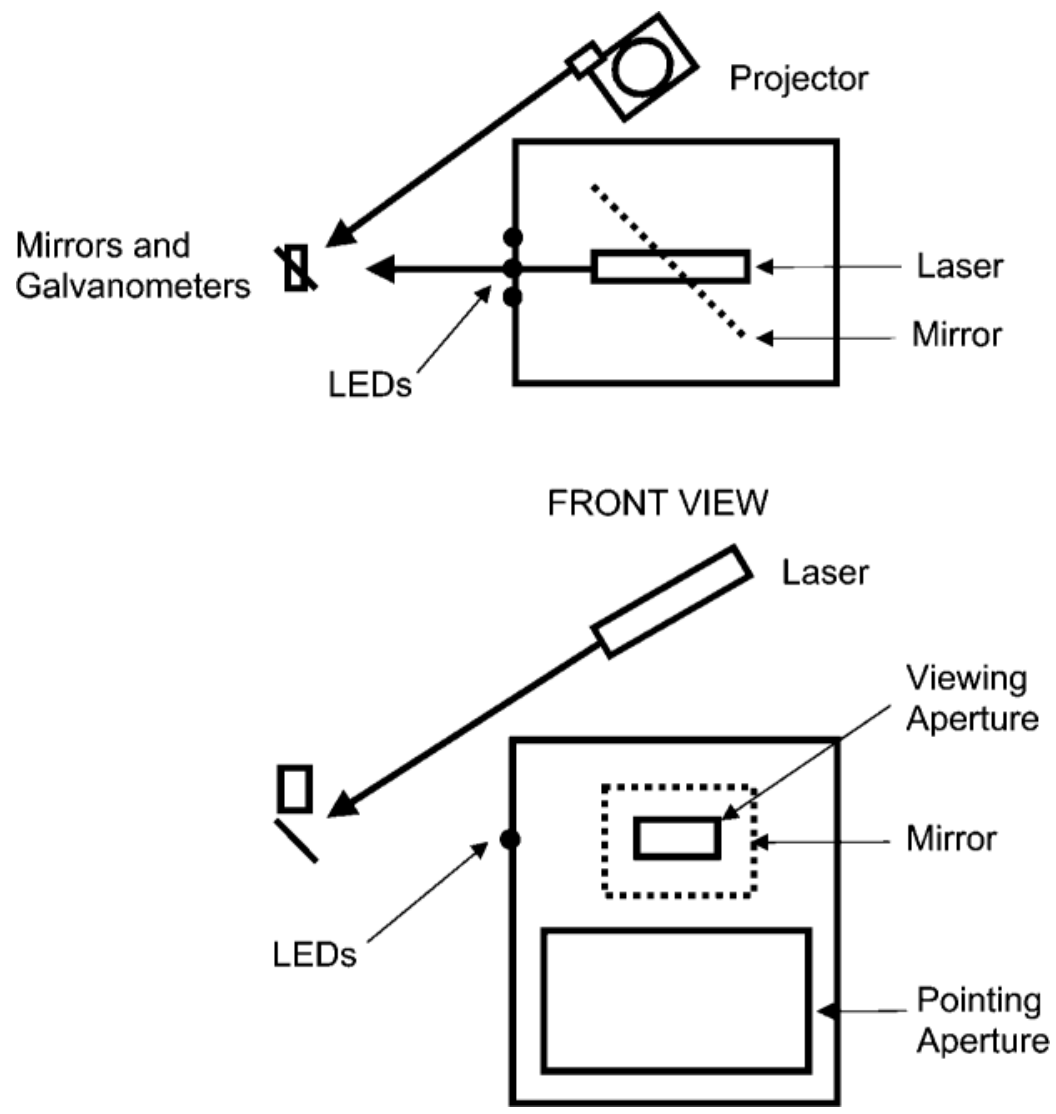

Figure 1. Schematic diagram of the apparatus. Top and front views are shown in the top and bottom portions of the figure, respectively. 


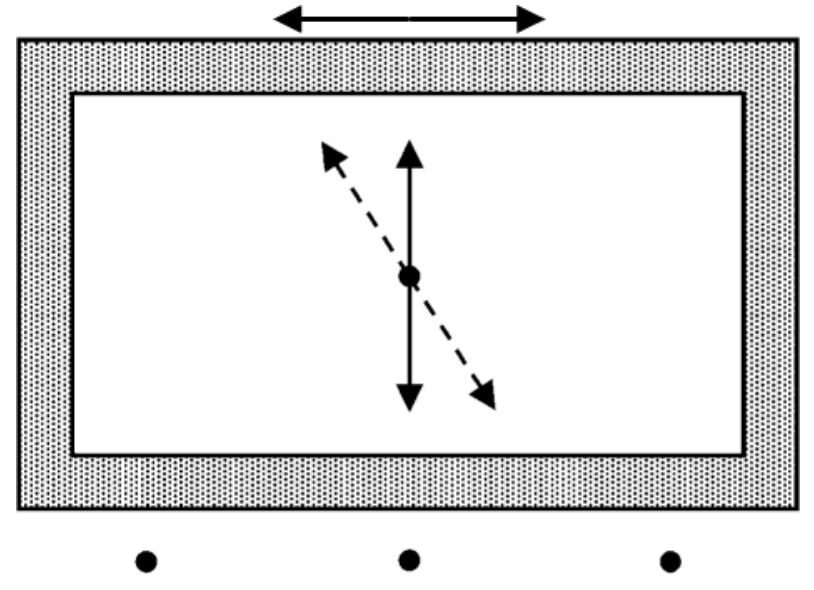

Figure 2. Schematic of the stimulus. A central dot stimulus oscillated vertically while a surrounding rectangular frame underwent phase-locked horizontal motion. Upward and downward dot motions were paired with rightward and leftward frame motion, respectively (solid arrows). The resulting motion path was on a slant tilted top-leftward as a result of IM (dashed arrows). Three LEDs below the frame could be flashed briefly.

puter control regulated the presentation of the dot and the rectangle. Three light-emitting diodes (LEDs) were positioned $1 \mathrm{~cm}$ below the lower edge of the rectangle and were separated by $5 \mathrm{~cm}$. The central LED was positioned directly below the laser spot. Each LED could also be briefly illuminated under microcomputer control. A rod, out of sight of the subject on the right side of the exterior of the box, was mounted so that its orientation could be adjusted in the frontoparallel plane.

Procedure. The subjects participated in one experimental session, during which they underwent a calibration procedure followed by the main part of the experiment. During calibration, they were instructed to fixate the dot stimulus as it traversed various slanted paths in the frontoparallel plane and to judge both the apparent slant of the motion path and the endpoints of this apparent motion. Seven dot motion slants were used, corresponding to the vertical and tilts (top-left) of every $10^{\circ}$ between vertical and $60^{\circ}$. The order of orientations varied randomly across subjects. The subjects adjusted the orientation of the unseen rod so that its felt orientation matched the seen path of the dot. During these measures, the subject's forearm rested on a table surface, and the rod was grasped with the thumb on one side and the fingers on the other. Three of these responses were made for each slant, and their median was recorded for further analysis. The subjects indicated the apparent endpoints of dot motion by reaching out with a pen, held in the unseen right hand, and marking the apparent location of the dot on the back wall of the box at the locations at which they saw it reverse direction. Five marks were made for each of the endpoints for each slant. Five subjects performed the slant-matching procedure first and then the endpoint marking procedure, and the other 5 performed the tasks in the reverse order.

Following the calibration procedure, measurements of IM and apparent location were obtained. For these measures, the subjects were instructed to fixate the dot stimulus, which moved vertically while the rectangular surround was either stationary or oscillated horizontally in phase with the dot motion. The pairing of up-down dot motion with right-left frame motion produced apparent motion on a slant tilted top-left. On the slant-matching trials, the subjects viewed two complete cycles of dot motion and then rotated the unseen rod with the right hand to match the apparent slant of the dot's path, while continuing to view the moving dot. On the localization trials, they viewed two full cycles of dot motion and then marked the endpoints of dot motion during the next five cycles. Half the subjects performed the slant-matching procedure first, and the other half performed the endpoint marking first. For each task, measures were first obtained with the rectangular frame oscillating and then with the frame stationary.

\section{Results}

The median location of the five marks made for both endpoints on the open-loop pointing calibration task was determined, and the slant of the line connecting these

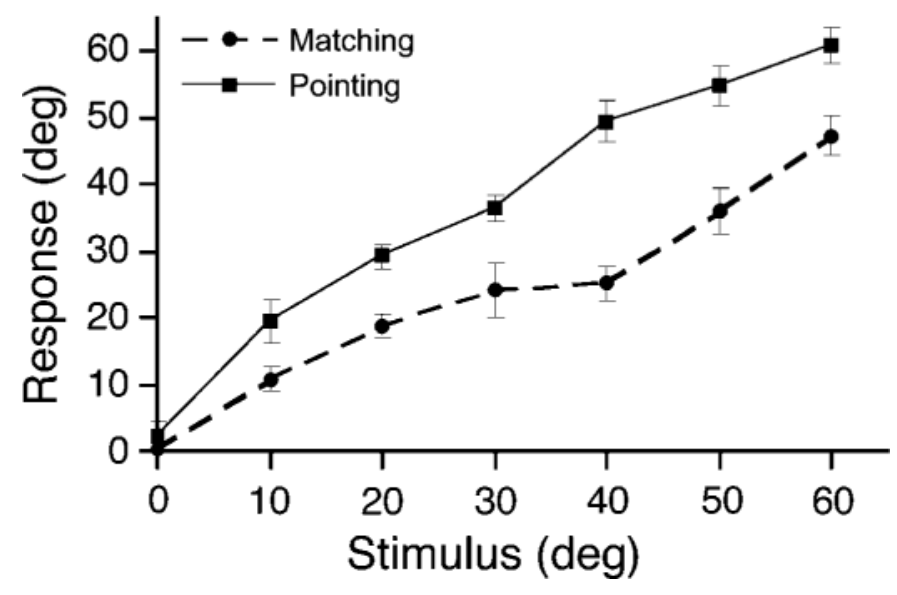

Figure 3. Mean response angle as a function of stimulus motion angle for both slant-matching and open-loop pointing calibration measures in Experiment 1 . Error bars correspond to $\pm 1 S E M$. Both measures were significantly correlated with stimulus angle $[F(1,58)=27.42, p<.0001$, and $F(1,58)=$ $116.15, p<.0001$, for the slant-matching and the open-loop pointing measures, respectively]. A small but statistically significant cubic component also contributed to the slant-matching measures $[F(1,58)=7.29, p<.01]$. 
scores was calculated and saved for further analysis. These derived slant measures are shown in Figure 3, together with the slant measures from the slant-matching calibration measures. It is apparent from the figure that different functions describe the results of the two procedures. The mean slope for the pointing measures was $0.706(S D=$ 0.15 ), with individual subjects' slopes ranging between 0.54 and 0.89 . The mean slope for the slant-matching measures was $0.953(S D=0.14)$, with individual subjects' slopes ranging between 0.68 and 1.11. Because the data showed differences between the measures and the actual stimulus slant, linear regression analyses were performed on both the slant-matching and the pointing calibration results for each subject. The obtained functions were then used to scale the results of the main part of the experiment. For example, if the regression of stimulus slant against a subject's slant-matching calibration measures produced a slope of 0.5 , the slant-matching measures obtained for that subject in the main part of the experiment were multiplied by .5 to yield "corrected" data. The mean scaling factor thereby attained for the pointing measures was $1.3(S D=$ 0.39 ), with individual subjects' scaling factors ranging between 0.85 and 2.16. The mean scaling factor for the slantmatching measures was $0.96(S D=0.11)$, with individual subjects' scaling factors ranging between 0.83 and 1.13 .

A given slant value represents the combination of a real vertical vector of dot motion $\left(6.6^{\circ}\right)$ with an illusory horizontal component created by IM. Thus, the illusory horizontal component of the slants was derived to reveal the magnitude of the IM effect (illusory horizontal vector = tangent (slant angle) $\times$ real vertical vector). For example, if the matched slant value was $45^{\circ}$, the horizontal illusory component was equal to the real vertical component of $6.6^{\circ}$. Measures obtained for each subject in the framestationary conditions were then subtracted from the corresponding measures in the frame-moving condition to yield the effect of frame motion. These computations were performed on both the raw and the scaled data, and the average results for all the subjects are shown in Figure 4 for both measures and both delay conditions. No effects of either measure or delay are apparent in the raw data shown in the top portion of the figure. In the scaled data in the bottom portion of the figure, however, it appears that there is a greater effect of frame motion on the slant-matching responses than on open-loop pointing responses and that the measures taken after the 5-sec delay are slightly smaller than those obtained immediately.

The results shown in the top portion of Figure 4 were analyzed with a 2 (response measure) $\times 2$ (delay condition) analysis of variance (ANOVA), which showed no significant main effects or interactions. However, a separate ANOVA conducted on the scaled results shown in the bottom portion of Figure 4 showed a statistically significant effect for response measure $[F(1,9)=7.84, p<.05]$. Neither delay condition $[F(1,9)=3.83, p=.08]$ nor its interaction with response measure $[F(1,9)=0.07, p>$ $.50]$ was statistically significant.
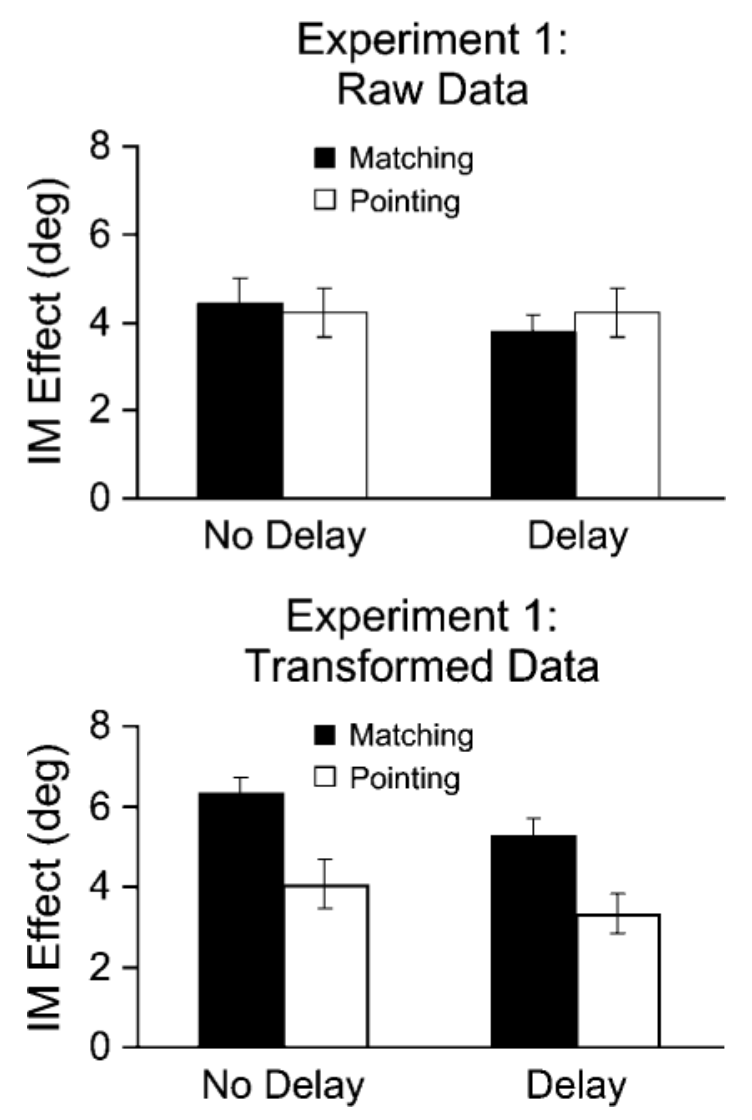

Figure 4. Mean effect of IM on slant-matching and open-loop pointing responses in the horizontal dimension in Experiment 1. Raw and transformed data are presented in the top and bottom portions of the figure, respectively. Error bars correspond to \pm 1 SEM.

\section{Discussion}

The primary finding of Experiment 1 was that IM had a greater effect on slant-matching judgments than on openloop pointing at the endpoints of the apparent motion path of the dot stimulus. The IM effect in the slant-matching/ no-delay data was $6.3^{\circ}$, essentially the same as the magnitude of frame motion $\left(6.6^{\circ}\right)$. Therefore, the gain of the IM, expressed as IM vector/frame motion, is approximately unity. The corresponding gain for the pointing/no-delay results was .61. This finding is consistent with several previous reports that IM has relatively less influence on localization measures, such as open-loop pointing, than one might expect from the apparent motion (Abrams \& Landgraf, 1990; Bacon et al., 1982; Bridgeman et al., 1981). In the present research, this pattern of results was extended to the vector-sum IM stimulus. In addition, the use of the calibration procedure precluded the possibility that any differences between the slant-matching and the open-loop pointing measures could be attributed to systematic errors in the slant-matching measure. It should also be noted that the use of the calibration procedure revealed a less appar- 
ent effect of IM on open-loop pointing than would have been concluded in its absence. That is, the calibration procedure made the two measures less commensurate with one another. Had it not been employed, it would have been erroneously concluded from the raw data that the effects of IM on slant-matching and pointing responses were not significantly different.

In the present research, pointing responses were always directed at the terminal location of the tracked IM stimulus, and little apparent effect of IM was obtained. In distinction, Sheth and Shimojo (2000) had subjects direct localization responses to either the initial or the terminal perceived locations of a target undergoing IM. Although there was little effect of IM on marking perceived terminal locations (as in the present study), there was a significant influence of IM on marking the initial (remembered) locations.

It is interesting that a delay between stimulus viewing and responding did not influence the magnitude of the IM effects on pointing, in contrast to the previously mentioned results in Mack, Heuer, Villardi, and Chambers (1985) for the Müller-Lyer illusion and in Bridgeman et al. (1997) for the Dietzel-Roelofs effect. A similar result in the present research would presumably cause pointing responses to be more biased in the direction of seen motion after a delay than with no delay. However, the present results nearly achieved a statistically significant effect in the opposite direction. In a prior study by Abrams and Landgraf (1990), both perceived motion and open-loop pointing were influenced by surround motion both with and without a delay. In that study, however, the delay manipulation occurred between experiments, and no statistical comparison of the effects of the delay was reported. Similarly, Sheth and Shimojo (2000) demonstrated that the difference in results for marking the perceived initial and terminal locations of an IM stimulus was not the consequence of a greater delay involved in the marking of the initial stimulus location.

Bridgman et al. (1997) have argued that the changes observed after a delay in open-loop pointing with the Roelofs effect are consistent with the conceptualization that the illusion is subject to vision-action dissociation. Specifically, it is proposed that the cognitive and motor maps initially have a representation of the location of the Roelofs target. The cognitive representation is incorrect (hence, the illusion), but the motor representation is more accurate (hence, more accurate pointing). The motor representation fades rapidly following stimulus offset, forcing the motor system to rely on the cognitive map after a delay, which results in open-loop pointing errors consistent with the illusion. A similar argument may be applied to the changes observed following a delay with the Müller-Lyer illusion. The present failure to obtain a delay effect on IM open-loop pointing suggests that this argument does not apply to IM, and at least in this regard, IM does not resemble other purported examples of vision-action dissociation. ${ }^{4}$

Alternatively, it might be argued that the no-delay condition in the present research involved a delay after all, in that the subjects experienced IM in the same direction for $5 \mathrm{sec}$ during the final half-cycle of stimulus motion before the stimulus was extinguished. Thus, the "immediate" pointing responses occurred after a delay of $5 \mathrm{sec}$ following the onset of IM. However, this is probably a moot point, because it is impossible to have a true no-delay condition, owing to the fact that IM requires a period of time to occur.

\section{EXPERIMENT 2}

Experiment 1, like several prior studies (Abrams \& Landgraf, 1990; Bacon et al., 1982; Bridgeman et al., 1981), demonstrated that surround motion influences the apparent location of a target undergoing IM. Experiment 2 was conducted to determine whether the localization effects of surround motion were the result of an alteration of either registered trunk or eye position. That is, it is possible that the surround motion causes subjects to misperceive the direction in which either their trunks or their eyes are oriented (McConkie \& Farber, 1979; Rock et al., 1980), which in turn alters pointing responses directed toward the fixated IM target. Thus, if observers perceive their eyes to be pointing to the left of true direction, their pointing will err to the left of the target. Alternatively, this same pointing error would occur if observers misperceive their trunk to be turned to the right relative to the head. In Experiment 2, pointing measures were obtained for both the dot stimulus and other targets that were flashed briefly following extinction of the IM display. It was reasoned that if IM caused either misregistered trunk or eye position, pointing responses to any visual stimulus would be altered in the direction of the immediately preceding IM, and by the same amount.

Experiment 2 was conducted in two phases. In the initial phase (Experiment 2A), data were collected from 8 subjects without monitoring eye movements. In the subsequent phase (Experiment 2B), 9 subjects participated in procedures identical to those in Experiment 2A, with the exception that eye movements were monitored and the subjects were instructed to maintain fixation at the location of the IM target. This condition was included to preclude the possibility that the subjects in Experiment 2A had saccaded their eyes to the briefly flashed LED target prior to marking its apparent location. Such a saccade might, in turn, have interfered with any effects on openloop pointing that the IM would otherwise have had (Nemire \& Bridgeman, 1987). Because of this concern that eye position registration may have been altered by saccades to the LED target, Experiment 2B removed this potential confound.

\section{Method}

Subjects. In Experiment 2A, 3 male and 5 female volunteers, 21-46 years of age, served as subjects. All had normal vision and either were capable of focusing at the display distance without correction or wore contact lenses. Two of the subjects were familiar with the experimental hypotheses. In Experiment 2B, 9 volunteers, 4 males and 5 females, $21-46$ years of age, served as subjects. Three 
of the subjects ( 2 males and 1 female) also had participated in Experiment $2 \mathrm{~A}$. All had normal vision and either were able to focus at the distance of the displays without correction or wore contact lenses. Two subjects were familiar with the hypotheses under investigation.

Apparatus. The viewing apparatus and displays were identical to those in Experiment 1. For the Experiment 2B measures, the chinrest was replaced with a bite bar. Horizontal and vertical eye movements were recorded with an Ober 2 system (Permobil Meditech, Inc.), which uses infrared detectors mounted on goggles attached to the subject's head with a strap. The goggles did not alter the subject's view of the stimulus display.

Procedure. In Experiment 2A, the subjects participated in a single testing session, during which they marked on separate blocks of trials the apparent locations of either the endpoints of motion of the fixation dot or one of three briefly flashed LED targets (see Figure 1). Before each trial, the experimenter announced that the trial was about to start. Then the stationary frame and the fixation dot appeared, and the subjects were instructed to fixate the dot. After approximately $1 \mathrm{sec}$, the frame and the dot began to oscillate in the same manner as that in Experiment 1. After 2.25 cycles, when the dot was at either its highest or its lowest point of excursion and the frame was in its rightmost or leftmost position, respectively, the entire stimulus array was extinguished, and the subjects made the pointing response. The two conditions of the display at stimulus offset are shown in Figure 5.

Fixation stimulus trials. On these trials, the subjects were instructed to reach out as quickly as possible with a pen and mark the place on the back wall of the apparatus where the target dot appeared to be just before it and the frame were extinguished. Each trial began with the subject's right hand resting near the body on the desktop that supported the apparatus. On six trials, the dot and the frame disappeared when the dot was at its highest excursion point and the frame in its far-right position. On the other six trials, initial dot motion was downward and the dot and frame extinguished at the lowest point of the dot's excursion, the frame having moved to its farleft position. The direction of offset was counterbalanced across subjects. A similar set of 12 control trials (6 top-offset/frame-right and 6 bottom-offset/frame-left) occurred with both the dot and the frame stationary at their terminal positions (dot-top/frame-right or dot-bottom/frame-left). The dot and the frame were presented for the same period of time prior to extinction for these stationary frame trials, as in the moving frame trials.

LED stimulus trials. The procedure for these trials was the same as that for the fixation stimulus trials until the dot and the frame were extinguished. At that point, a delay of $14 \mathrm{msec}$ occurred, after which one of the three LEDs situated beneath the frame (see Figure 1) appeared for $50 \mathrm{msec}$ and the subject marked its apparent location with the pen as quickly as possible. There were 36 of these trials, 6 for each of the three LED stimuli following the offset of the frame and the dot, when the dot was at the lowest point of its motion and the frame at the extreme left position, and a corresponding set of 18 trials following offset of the dot at its highest point and the frame in its extreme right position. Thirty-six control trials (18 top-offset/frameright and 18 bottom-offset/frame-left) occurred with the dot and

\section{Offset: Dot top/frame right}

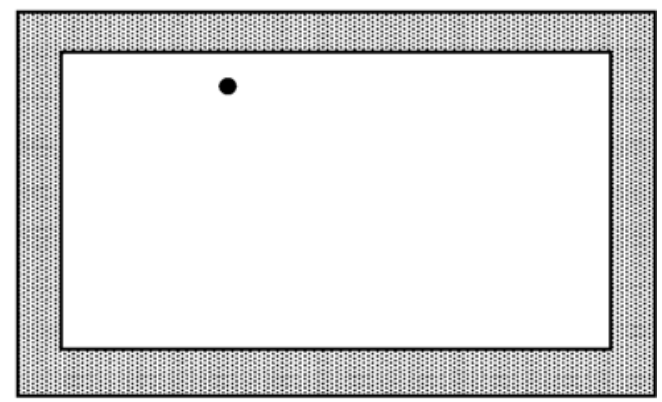

Offset: Dot bottom/frame left

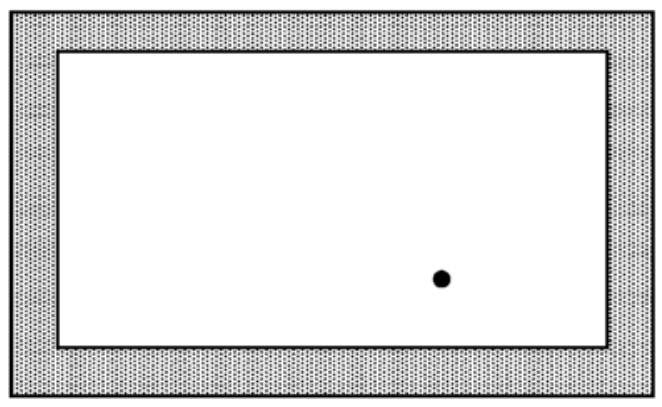

Figure 5. Stimulus display at offset in Experiment 2 prior to pointing responses. Top portion: display offset when the tracked dot was at the top of its path and the frame was at the far right extent of its motion. Bottom portion: display offset when the tracked dot was at the bottom of its path and the frame was at the far-left extent of its motion. Note that (despite appearances) the dot is displayed in the same horizontal position for the two offset conditions. 
frame stationary. Because the briefly flashed LED targets appeared only after the target dot and the frame were extinguished, they were never subject to IM.

Half of the subjects completed the fixation stimulus trials before the LED stimulus trials, and the other half completed the two types of trials in the reverse order. Within a given block of trials, half of the subjects performed the moving frame trials first, and half performed the stationary frame trials first. Within the LED stimulus trials, the order in which the three LEDs were presented differed for each subject.

In Experiment 2B, the procedure was similar to that in Experiment $2 \mathrm{~A}$, with the primary difference that the subjects were required to maintain eye position at the perceived location of the fixation target when it was extinguished while making localization responses for all the targets. The subjects' eye movements were monitored to ensure compliance with this instruction. Prior to the start of the experiment, the subjects made a series of saccades to LEDs displayed at $5^{\circ}$ intervals $\left( \pm 10^{\circ}\right)$ along the horizontal and vertical axes. The subjects viewed each of these targets for approximately $3 \mathrm{sec}$ prior to the next saccade. Recordings of eye position were displayed on the computer monitor, and the position of the goggles and the gain of the system were adjusted to enhance sensitivity. All the recordings were made at $120 \mathrm{~Hz}$.

On each trial, horizontal and vertical eye movement traces were displayed on the computer monitor and were viewed by the experimenter. The subjects who failed on a given trial to obey the fixation instructions by making a saccade were told to cease moving the pointing hand before the perceived location could be marked, and the trial was repeated. Before the experiment began, the subjects were given a block of 10 practice trials, followed by another set if it was deemed necessary. Two subjects were unable to comply with the instructions, consistently making eye movements to the LED targets. The data from these 2 subjects were excluded.

\section{Results}

The median location on the $x$-axis of the six marks for the fixation dot and each of the three LEDs was calculated for each of the four combinations of frame motion (moving vs. stationary) and stimulus offset (top-offset/frameright vs. top-offset/frame-left). These values were then used in all further analyses. The actual location on the horizontal axis of each target was then subtracted from the median values to yield error scores. Positive and negative values represented pointing errors to the right and the left of actual target location, respectively. The mean error scores for the frame-stationary control trials from Experiments $2 \mathrm{~A}$ and $2 \mathrm{~B}$ are presented in the top and bottom portions, respectively, of Figure 6 . It is apparent that mean pointing errors tended to be to the left of the left LED and to the right of the right LED, with little systematic pointing error for either the central LED or the IM target in both experimental phases. The results shown in the top and bottom portions of Figure 6 were analyzed by separate 2 (frame direction, right/left) $\times 4$ (target) ANOVAs. For Experiment $2 \mathrm{~A}$, the main effect of target was statistically significant $[F(3,21)=7.82, p<.01]$, as was the interaction of frame direction and target $[F(3,21)=12.68, p<$ $.001]$. The main effect of frame direction was not statistically significant. Planned comparisons indicated that the mean errors for both the left and the right LEDs differed significantly from those for the central $\operatorname{LED}[t(7)=4.69$, $p<.01$, and $t(7)=4.94, p<.01$, respectively], and there
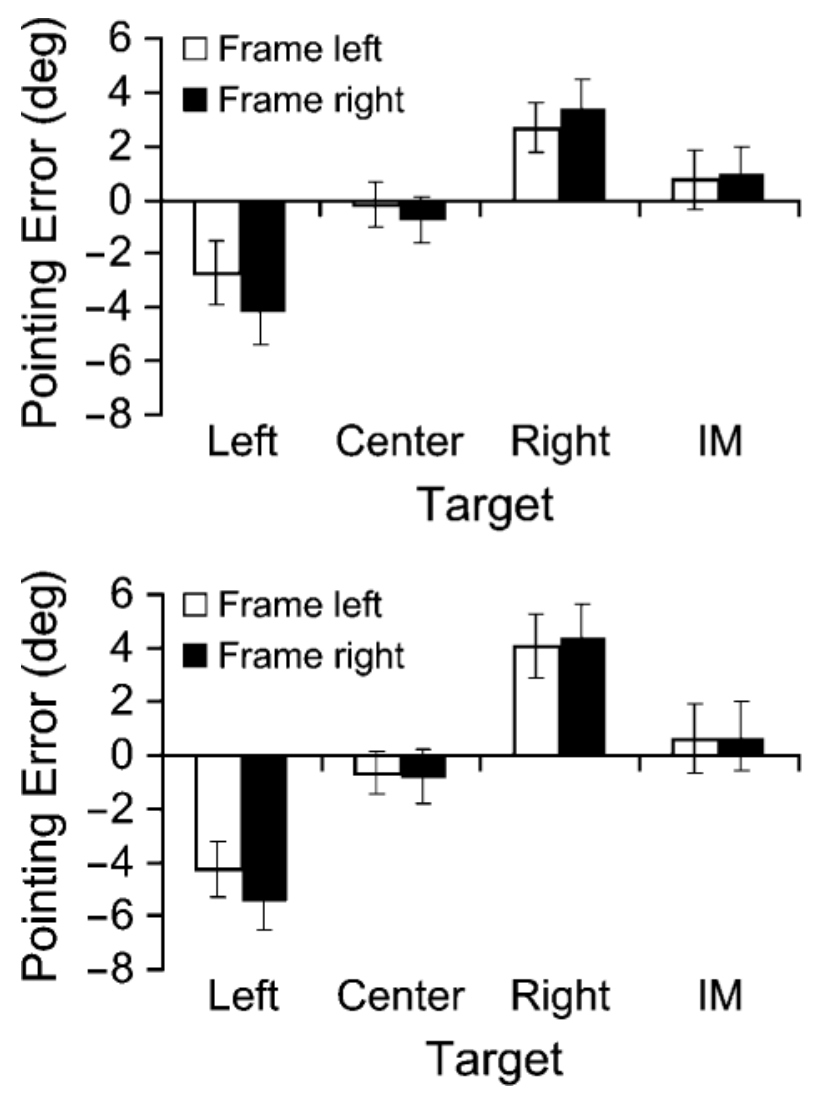

Figure 6. Results from Experiment 2A (top portion) and 2B (bottom portion). Plotted values represent mean pointing errors in the horizontal dimension for all targets obtained on framestationary trials for both directions of frame offset. Pointing errors to the right and left are plotted as positive and negative values, respectively. Error bars correspond to \pm 1 SEM. IM, induced motion.

was no significant difference between the results for the central LED and the IM target $[t(7)=1.62, p=.15]$. The significant interaction appears to consist of a greater difference between the results for the left and the right LEDs with the frame offset to the right than for those with the frame offset to the left. A planned comparison supported this conclusion $[t(7)=4.77, p<.01]$. For the Experiment $2 \mathrm{~B}$ results, the main effect of target was statistically significant $[F(3,21)=30.452, p<.001]$. Neither the main effect of frame direction nor the interaction of frame direction and target was statistically significant. Planned comparisons indicated that the mean errors for both the left and the right LEDs differed significantly from those for the central $\operatorname{LED}[t(6)=4.58, p<.01$, and $t(6)=9.78$, $p<.001$, respectively], and there was no significant difference between the results for the central LED and those for the IM target $[t(6)=1.96, p=.10]$.

For each target, the error score obtained with the frame stationary (Figure 6) was subtracted from the corresponding error score obtained with the frame moving for both top-offset/frame-right and bottom-offset/frame-left con- 
ditions. Positive values were assigned to instances in which the location with frame motion was marked to the right of the location with the frame stationary. These difference scores, which reveal the effect of frame motion on apparent location, are shown for all four targets for both offset conditions in Figure 7. The results from Experiments $2 \mathrm{~A}$ and $2 \mathrm{~B}$ are shown in the top and bottom portions, respectively, of the figure. It can be seen that, following rightward and leftward frame motion, the IM target is marked as being to the left and the right, respectively, of its apparent location during the control measures with a stationary target and frame. A similar, but smaller, tendency may be present in the results for the LED targets as well.

The results shown in the top and bottom portions of Figure 6 were analyzed by separate 2 (frame direction, right/left) $\times 4$ (target) ANOVAs. For Experiment 2A, the main effect of frame direction was statistically significant $[F(1,7)=10.41, p<.05]$, as was the interaction of frame direction and target $[F(3,21)=12.68, p<.001]$. The

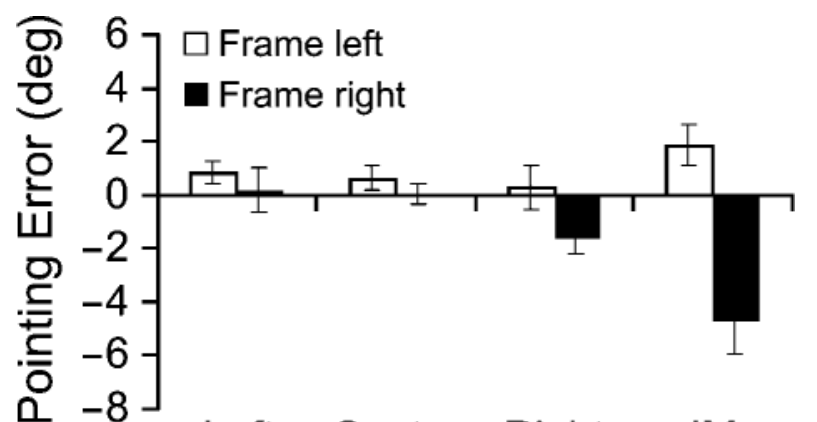

\section{Left Center Right IM Target}

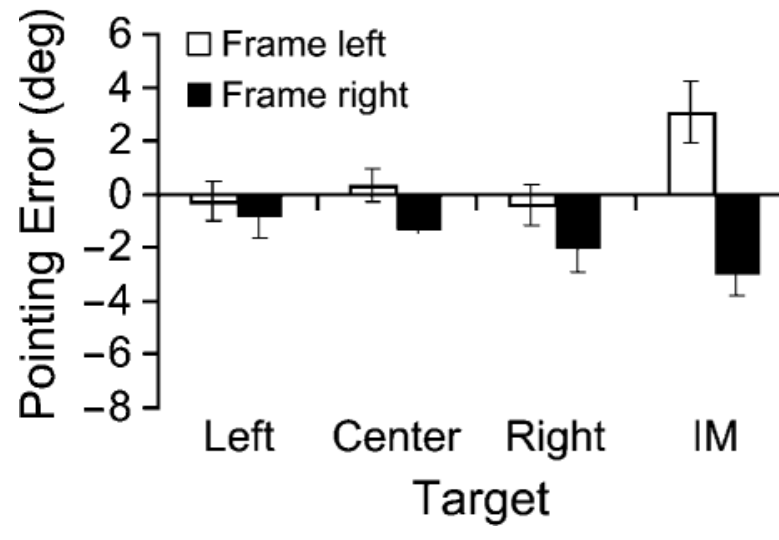

Figure 7. Results from Experiment 2A (top portion) and 2B (bottom portion). Effects of frame motion on pointing errors in the horizontal dimension are shown for each of the four targets. Plotted values represent mean pointing errors obtained on the frame motion trials minus corresponding mean pointing errors on the frame-stationary trials. Pointing errors to the right and left are plotted as positive and negative values, respectively. Error bars correspond to $\pm 1 S E M$. IM, induced motion. main effect of target was not statistically significant. Planned comparisons indicated that the effect of frame direction was statistically significant for the case of the IM target $[t(7)=4.82, p<.01]$, but there was no statistically significant effect of frame direction on the results for any of the three LED targets in isolation $[t(7)=0.74, p=.48$, $t(7)=1.01, p=.38$, and $t(7)=1.77, p=.12$, respectively]. However, when all three LEDs were combined, the effect of frame direction was statistically significant $[t(23)=2.10, p<.05]$. For Experiment $2 \mathrm{~B}$, the main effect of frame direction was statistically significant $[F(1,6)=$ $14.26, p<.01]$, as was the interaction of frame direction and target $[F(3,18)=6.16, p<.01]$. The main effect of target was not statistically significant. Planned comparisons indicated that the effect of frame direction was statistically significant for the case of the IM target $[t(6)=$ $3.57, p<.05]$, but there was no statistically significant effect of frame direction on the results for any of the three LED targets in isolation $[t(6)=0.89, p=.48, t(6)=1.95$, $p=.10$, and $t(6)=2.03, p=.09$, respectively]. However, a planned comparison of the effect of frame direction on the three LED targets combined was statistically significant $[t(20)=2.94, p<.01]$.

\section{Discussion}

The analysis of the stationary frame data (Figure 6) failed to demonstrate an effect on open-loop pointing of frame location at offset. This is of interest because this stimulus configuration represents the Dietzel-Roelofs effect, in which the surrounded dot is mislocalized in the direction opposite the displacement of the frame (e.g., Bridgeman et al., 1997; Bruell \& Albee, 1955; Dietzel, 1924; Roelofs, 1935). This illusion, which the reader can experience in Figure 5, has been proposed as the basis for IM (Bridgeman \& Klassen, 1983; Brosgole, 1968). The absence of pointing errors despite the presence of the illusion is consistent with the report of Bridgeman et al. (1997) that this configuration affects perceptual or cognitive measures, but not open-loop pointing.

The stationary frame data also revealed that pointing responses aimed at the left and the right LEDs were systematically biased toward locations more eccentric than the LEDs. That is, mean pointing responses were to the left of the left LED and to the right of the right LED. This pattern appears to replicate other reports of a peripheral displacement effect (e.g., Bock, 1986; Bock \& Daunicht, 1987; Gillam \& Chambers, 1985; Mack, Heuer, Villardi, \& Chambers 1985; Tsal \& Bareket, 1999; Tsal, Meiran, \& Lamy, 1995; Welch, Post, Lum, \& Prinzmetal, in press).

The analysis of the moving frame data (Figure 7) confirms the finding of Experiment 1 and other reports (Abrams \& Landgraf, 1990; Bacon et al., 1982; Bridgeman et al., 1981) that a target seen to undergo IM is also mislocalized in the direction of the perceived motion. In addition, individual targets flashed briefly following the observation of IM were not mislocalized in the direction of the IM target, contradicting the hypothesis that IM causes a misperception of either the position of the trunk 
or the direction of gaze. There was, however, a nonsignificant tendency for the individual LED targets to be mislocalized in the same direction as the IM target. When the results for all three LEDs were combined, this tendency was statistically significant. This small effect would be consistent with an influence of IM on either perceived gaze or trunk direction. However, the results also clearly demonstrate that this effect is too small to account for the mislocalization of the IM target.

\section{GENERAL DISCUSSION}

The results of Experiment 2 demonstrate that targets seen to undergo IM are subsequently mislocalized and that the effect of frame motion does not have a significant effect on pointing directed at other briefly flashed targets. The latter finding contradicts the hypothesis that IM causes a misregistration of trunk position, as well as the hypothesis that misperception of gaze direction generates the pointing errors (McConkie \& Farber, 1979; Rock et al., 1980; Soechting et al., 2001). In this regard, the findings are consistent with those of Mack, Heuer, Fendrich, et al. (1985) and, additionally, extend them to visual targets localized by means of open-loop pointing. Perhaps the most dramatic effect in this regard concerns the central LED, which, despite appearing directly below the IM target on the subject's median plane, was not mislocalized, whereas the IM target was. Neither of the auditory targets in the study by Mack, Heuer, Fendrich, Vilardi, and Chambers (1985) was aligned with the IM target.

The present pattern of results is, in some ways, similar to the results of Hansen (1979). In that study, it was shown that during pursuit of a moving stimulus, the apparent path of another untracked stimulus was systematically misperceived. Despite the illusion of path direction, the subjects were able to reliably make motor responses directed at the real locations of the unfixated stimulus.

The findings in this article that a target undergoing IM is mislocalized by a lesser amount than the observed IM replicates other reports (Abrams \& Landgraf, 1990; Bacon et al., 1982; Bridgeman et al., 1981). This difference between localization error and IM is surprising, however, only if one assumes that the perceptions of location and motion obey the rules of Euclidean space. That is, just as real object motion (or velocity) integrated over time yields a real displacement in space, it might be assumed that any seen motion (even if illusory) integrates into perceived displacement. The data clearly do not support this assumption. Rather, they suggest either an incomplete integration or some other explanation for why the IM target is mislocalized. Abrams and Landgraf as well as Smeets and Brenner (1995) have previously stressed the independence of location and motion information in IM.

The conclusion that the direction of gaze is not misregistered following IM does not preclude oculomotor contributions to IM. For instance, the nystagmus suppression hypothesis of IM (e.g., Post, 1986; Post \& Leibowitz, 1985) holds that IM is a result of activation of the pursuit system to oppose and cancel the involuntary tracking of the moving inducing stimulus that would otherwise occur. The result is no eye movement, even though the pursuit system's activation is perceptually registered as eye motion in the direction of pursuit effort. The pursuit system's signal corresponds to velocity, and there is no mechanism within the nystagmus suppression hypothesis that integrates this velocity signal into displacement. Therefore, no misregistration of gaze direction would be expected from this hypothesis.

If misregistration of neither trunk orientation nor gaze direction underlies the mislocalization of targets that have been seen to undergo IM, what does? One possibility is that offered by Mack, Heuer, Fendrich, et al. (1985), who suggested that it represents a form of response bias. That is, subjects, having seen a target move in a particular direction, bias their estimates of its position in the same direction in an attempt to be consistent. An alternate possibility is that the mislocalization reflects a property of the representation, or object file (Kahneman, Treisman, \& Gibbs, 1992), of the IM target. That is, in addition to such attributes as size, color, or velocity, it is possible that the representation of the IM target contains a location attribute as well, which is modified to a limited degree by prior seen motion. In this conceptualization, the IM target's mislocalization reflects a perceptual effect, rather than a response bias. This mislocalization, being a property of the representation of a particular stimulus, however, would not extend to other stimuli, either visual or auditory.

\section{REFERENCES}

Abrams, R. A., \& LANDGRaF, J. Z. (1990). Differential use of distance and location information for spatial localization. Perception \& Psychophysics, 47, 349-359.

Aglioti, S., DeSouza, J. F. X., \& Goodale, M. A. (1995). Size-contrast illusions deceive the eye but not the hand. Current Biology, 5, 679-685.

Bacon, J. H., Gordon, A., \& Schulman, P. H. (1982). The effect of two types of induced-motion displays on perceived location of the induced target. Perception \& Psychophysics, 32, 353-359.

Bassili, J. N., \& Farber, J. M. (1977). Experiments on the locus of induced motion. Perception \& Psychophysics, 21, 157-161.

Binsted, G., Chua, R., Helsen, W., \& Elliott, D. (2001). Eye-hand coordination in goal-directed aiming. Human Movement Science, 20, 563-585.

BiNSTED, G., \& ElLIOTt, D. (1999). Ocular perturbations and retinal/extraretinal information: The coordination of saccadic and manual movements. Experimental Brain Research, 127, 193-206.

Bock, O. (1986). Contribution of retinal versus extraretinal signals towards visual localization in goal-directed movements. Experimental Brain Research, 64, 467-482.

BocK, O., \& DAUNICHT, W. J. (1987). Information processing in goaldirected movements. Behavior \& Brain Research, 23, 23-28.

Bridgeman, B., KirCh, M., \& Sperling, A. (1981). Segregation of cognitive and motor aspects of visual function using induced motion. Perception \& Psychophysics, 29, 336-342.

Bridgeman, B., \& Klassen, H. (1983). On the origin of stroboscopic induced motion. Perception \& Psychophysics, 34, 149-154.

Bridgeman, B., Peery, S., \& Anand, S. (1997). Interaction of cognitive and sensorimotor maps of visual space. Perception \& Psychophysics, 59, 456-469.

Brosgole, L. (1968). An analysis of induced motion. Acta Psychologica, 28, 1-44.

Brosgole, L., Cristal, R. M., \& Carpenter, O. (1968). The role of 
eye movements in the perception of visually induced motion. Perception \& Psychophysics, 3, 166-168.

Bruell, J. H., \& AlbeE, G. W. (1955). Effect of asymmetrical retinal stimulation on the perception of the median plane. Perceptual \& Motor Skills, 5, 133-139.

Cohen, Y. E., \& Andersen, R. A. (2000). Reaches to sounds encoded in an eye-centered reference frame. Neuron, 27, 647-652.

Creem, S. H., \& ProffitT, D. R. (1998). Two memories for geographical slant: Separation and interdependence of action and awareness. Psychonomic Bulletin \& Review, 5, 22-36.

DiETZEL, H. (1924). Untersuchungen über die optische Lokalisation der Mediane. Zeitschrift für Biologie, 80, 289-316.

DunCKER, K. (1929). Über induzierte Bewegung [On induced motion]. Psychologische Forschung, 12, 180-259.

Elliott, D., \& LeE, T. D. (1995). The role of target information on manual-aiming bias. Psychological Research, 59, 2-9.

Gentilucci, M., Chieffi, S., Daprati, E., Saetti, M. C., \& Toni, I. (1996). Visual illusion and action. Neuropsychologia, 34, 369-376.

Gillam, B., \& Chambers, D. (1985). Size and position are incongruous: Measurements on the Müller-Lyer figure. Perception \& Psychophysics, 37, 549-556.

GLOVER, S. R., \& Dixon, P. (2001). Dynamic illusion effects in a reaching task: Evidence for separate visual representations in the planning and control of reaching. Journal of Experimental Psychology: Human Perception \& Performance, 27, 560-572.

Gogel, W., \& MACCRACKen, P. J. (1979). Depth adjacency and induced motion. Perceptual \& Motor Skills, 48, 343-350.

HafFenden, A. M., \& GoOdAle, M. A. (1998). The effect of pictorial illusion on prehension and perception. Journal of Cognitive Neuroscience, 10, 122-136.

HANSEN, R. M. (1979). Spatial localization during pursuit eye movements. Vision Research, 19, 1213-1221.

HARRIS, C. S. (1974). Beware of the straight-ahead shift-a nonperceptual change in experiments on adaptation to displaced vision. Perception, 3, 461-476.

Hu, Y., \& GoOdALE, M. A. (2000). Grasping after a delay shifts sizescaling from absolute to relative metrics. Journal of Cognitive Neuroscience, 12, 856-868.

KAHNEMAN, D., Treisman, A., \& GibBs, B. J. (1992). The reviewing of object files: Object-specific integration of information. Cognitive Psychology, 24, 175-219.

LoOmis, J. M., DA Silva, J. A., Fujita, N., \& Fukusima, S. S. (1992). Visual space perception and visually directed action. Journal of Experimental Psychology: Human Perception \& Performance, 18, 906-921.

MACK, A., FENDrich, R., \& Wong, E. (1982). Is perceived motion a stimulus for smooth pursuit? Vision Research, 22, 77-88.

Mack, A., Heuer, F., Fendrich, R., Vilardi, K., \& Chambers, D. (1985). Induced motion and oculomotor capture. Journal of Experimental Psychology: Human Perception \& Performance, 11, 329-345.

Mack, A., Heuer, F., Villardi, K., \& Chambers, D. (19) sociation of position and extent in Müller-Lyer figures. Perception \& Psychophysics, 37, 335-344.

MCCONKIE, A. B., \& FARBER, J. M. (1979). Relation between perceived depth and perceived motion in uniform flow fields. Journal of Experimental Psychology: Human Perception \& Performance, 5, 501-508.

Milner, D., \& Goodale, M. (1995). The visual brain in action. Oxford: Oxford University Press.

Nemire, K., \& BRIDGEMAN, B. (1987). Oculomotor and skeletal motor systems share one map of visual space. Vision Research, 27, 393-400.

Post, R. B. (1986). Induced motion considered as a visually induced oculogyral illusion. Perception, 15, 131-138.

Post, R. B., \& Leibowitz, H. W. (1985). A revised analysis of the role of efference in motion perception. Perception, 14, 631-643.
Proteau, L., \& Masson, G. (1997). Visual perception modifies goaldirected movement control: Supporting evidence from a visual perturbation paradigm. Quarterly Journal of Experimental Psychology, 50A, 726-741.

Rock, I., Auster, M., Schiffman, M., \& Wheeler, D. (1980). Induced movement based on subtraction of motion from the inducing object. Journal of Experimental Psychology: Human Perception \& Performance, 6, 391-403.

RoELOFS, $\bar{C} .(1935)$. Optische localisation. Archives für Augenheilkunde, 109, 395-415.

Sheth, B. R., \& Shimojo, S. (2000). In space, the past can be recast but not the present. Perception, 29, 1279-1290.

SmeEts, J. B. J., \& BrenNer, E. (1995). Perception and action are based on the same visual information: Distinction between position and velocity. Journal of Experimental Psychology: Human Perception \& Performance, 21, 19-31.

Soechting, J. F., Engel, K. C., \& Flanders, M. (2001). The Duncker illusion and eye-hand coordination. Journal of Neurophysiology, $\mathbf{8 5}_{2}$ 843-854.

StricANnE, B., ANDERSEn, R. A., \& MAZzoni, P. (1996). Eye-centered, head-centered, and intermediate coding of remembered sound locations in area LIP. Journal of Neurophysiology, 76, 2071-2076.

Tsal, Y., \& BAREKET, T. (1999). Effects of attention on localization of stimuli in the visual field. Psychonomic Bulletin \& Review, $\underline{6}, 292-$ 296.

Tsal, Y., Meiran, N., \& Lamy, D. (1995). Toward a resolution theory of visual attention. Visual Cognition, 2, 313-330.

Wallach, H., Bacon, J., \& Schulman, P. (1978). Adaptation in motion perception: Alteration of induced motion. Perception \& Psychophysics, 24, 509-514.

WELCH, R. B., \& Post, R. B. (1996). Accuracy and adaptation of reaching and pointing in pitched visual environments. Perception \& Psychophysics, 58, 383-389.

Welch, R. B., Post, R. B., Lum, W., \& Prinzmetal, W. (in press). The relationship between perceived length and egocentric location in Müller-Lyer figures with one versus two chevrons. Perception \& Psychophysics.

Westwood, D. A., McEachern, T., \& Roy, E. A. (2001). Delayed grasping of a Müller-Lyer figure. Experimental Brain Research, $\mathbf{1 4 1}_{2}$ 166-173.

Wraga, M., Creem, S. H., \& Proffitt, D. R. (2000). Perception-action dissociations of a walkable Müller-Lyer configuration. Psychological Science, 11, 239-243.

\section{NOTES}

1. Subsequent to representation in head-centered coordinates, auditory stimuli may be remapped in ocular coordinates for the generation of saccades (Stricanne, Andersen, \& Mazzoni, 1996) or reaching movements (Cohen \& Andersen, 2000).

2. For additional recent work on the Müller-Lyer illusion's influence on hand movement, eye movement, and eye-hand coordination, see Binsted and Elliott (1999) and Binsted, Chua, Helsen, and Elliott (2001).

3. Placement of the back wall at the same optical distance as the display was intended to ensure that the reaching trajectory was interrupted at the approximate distance of its planned endpoint.

4. A reviewer has suggested that since the present study involved the tracking of a moving stimulus and the other cited examples of putative perception-action dissociation did not, it is possible that the differential results reflect the different neural signals available from tracking.

(Manuscript received February 24, 2003; revision accepted for publication November 11, 2003.) 